\title{
Trajectory Tracking Control of Deep Sea Mining Vehicle Based on Iterative Learning Algorithm
}

\author{
Yun Liu ${ }^{1, a^{*}}$,Shanshan Guo ${ }^{1, b}$ \\ ${ }^{1}$ College of Mechanical-Electrical and Vehicle Engineering, Weifang University Weifang, P.R.China \\ wflyun@126.com,lucy001478@163.com
}

Keywords: Deep-sea mining vehicle; Motion trajectory; Iterative learning

\begin{abstract}
In this paper, an open-loop and closed-loop iterative learning control algorithm based on iterative learning theory is proposed to study the working characteristics and control technology of deep-sea mining vehicles. In this method, the deviation of the heading angle and the trajectory deviation are taken as the feedback correction variables in each iteration, and the trajectory deviation of the desired trajectory is corrected continuously, so that the motion trajectory can be precisely controlled.
\end{abstract}

\section{Introduction}

Iterative learning control adopts the control strategy of "learning in repeated motion", which has the function of system memory and experience correction, and is applicable to the controlled object with repetitive operating characteristics[1-3]. Deep-sea mining vehicle in the seabed area for loading, transportation, mining and other actions, the work flow is fixed, especially during transportation, travel routes are relatively fixed and repeated, coincides with the characteristics of the controlled object in the theory of iterative learning. In order to improve the control precision of trajectory, a new algorithm of motion trajectory control for deep sea mining vehicle is proposed by using iterative learning control theory. By studying the control parameters in the iterative process, the accurate control of the trajectory can be achieved.

\section{Iterative learning Control Algorithm and its Basic Theory}

Iterative learning control attempts to control the controlled object by using the deviation between the output trajectory and the given trajectory as the feedback to correct the suboptimal control signal, therefore new control signals are generated, so that the tracking control performance of the system is improved[4-6].

The dynamic model of the controlled system is as follows[7] :

$$
\left\{\begin{array}{l}
\dot{x}(t)=f[t, \chi(t), u(t)] \\
y(t)=g[t, \chi(t), u(t)]
\end{array}\right.
$$

Where $x \subset R^{n \times 1}$ is state vector, $y \subset R^{m \times 1}$ is output vector, $u \subset R^{r \times 1}$ is the control vector of the controlled system, $\mathrm{f}$ and $\mathrm{g}$ are functions with corresponding dimension, and the structure and parameters are unknown. The output $y(t)$ of the system in the time domain $t \in[0, T]_{\text {is required to }}$ track the desired output $y_{r}(t)$ as much as possible, assuming the desired control vector $u_{r}(t)$ is present. That is, under the given initial state value ${ }^{x(0)}, u_{r}(t)$ is the solution of Eq. 1 when $y(t)=y_{r}(t)$. The goal of iterative learning control is to operate repeatedly, under certain learning laws to make $u(t) \rightarrow u_{r}(t), y(t) \rightarrow y_{r}(t)$. In the $\mathrm{k}$ run, Eq. 1 is indicated as:

$$
\left\{\begin{array}{l}
\dot{x}_{k}(t)=f\left[t, x_{k}(t), u_{k}(t)\right] \\
y_{k}(t)=g\left[t, x_{k}(t), u_{k}(t)\right]
\end{array}\right.
$$

The tracking error is indicated as: 
$e_{k}(t)=y_{r}(t)-y_{k}(t)$

The subscript $\mathrm{k}$ in the form represents the $\mathrm{k}$ run, assuming that the expected output $y_{r}(t)$ remains constant during each run.

\section{Open loop and Closed Loop Iterative Learning Control Algorithm}

Iterative learning control can be divided into two kinds: open-loop iterative learning and closed-loop iterative learning. There are many kinds of learning laws, the PID type learning law is adopted in this paper[8].

\section{Open-loop control algorithm}

The open-loop iterative learning control algorithm is shown as Eq.4. The control quantity at $\mathrm{k}+1$ equals to the control quantity of the $\mathrm{k}$, plus the linear combination of proportions, integrals, and differential terms at $\mathrm{k}$ output error, that is:

$$
u_{k+1}(t)=u_{k}(t)+K_{p} e_{k}(t)+K_{i} \int_{0}^{t} e_{k}(s) d s+K_{d} \frac{d e_{k}(t)}{d t}
$$

Where $K_{p}, K_{i}, K_{d}$ are scale, integral and differential coefficients respectively. The basic structure of the open-loop iterative learning control algorithm is shown in Fig. 1.

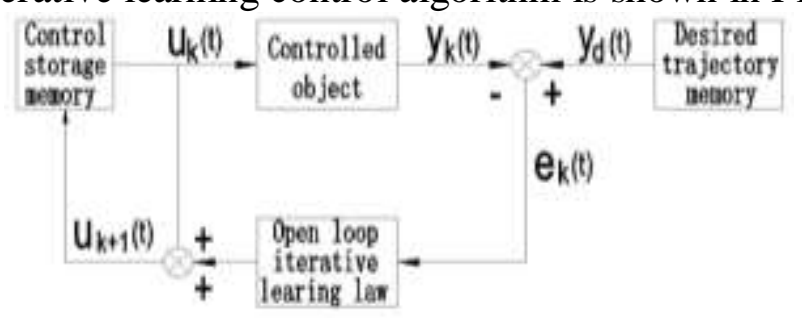

Figure 1. Block Diagram of Open Loop Iterative Learning Control Algorithm

General open-loop iterative learning control algorithms can be written as:

$$
u_{k+1}(t)=L\left[u_{k}(t), e_{k}(t)\right]
$$

Where $\mathrm{L}$ is linear or nonlinear operator.

\section{Closed-loop control algorithm}

The closed-loop iterative learning control algorithm is shown as Eq.6, taking the error of the $\mathrm{k}+1$ run process as a feedback correction, that is:

$$
u_{k+1}(t)=u_{k}(t)+K_{p} e_{k+1}(t)+K_{i} \int_{0}^{t} e_{k+1}(s) d s+K_{d} \frac{d e_{k+1}(t)}{d t}
$$

Where $K_{p}, K_{i}, K_{d}$ are scale, integral and differential coefficients respectively. The basic structure of the closed-loop iterative learning control algorithm is shown in Fig. 2.

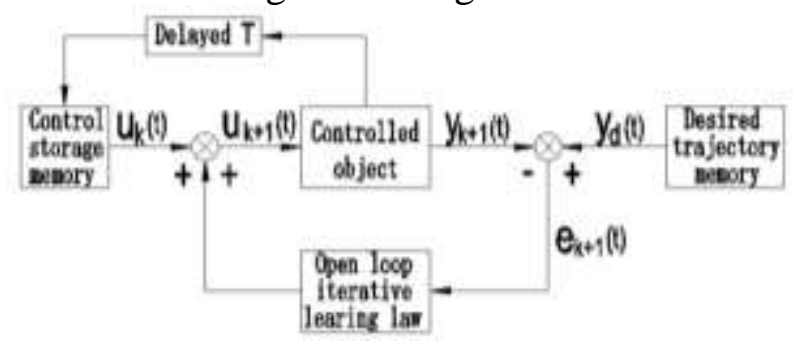

Figure 2. Block Diagram of Closed Loop Iterative Learning Control Algorithm

General closed-loop iterative learning control algorithms can be written as:

$$
u_{k+1}(t)=L\left[u_{k}(t), e_{k+1}(t)\right]
$$


Where $\mathrm{L}$ is linear or nonlinear operator.

\section{Discretization of Iterative Learning Control Algorithm}

The open loop and closed loop control algorithms are discretized respectively, and the Eq. 1 is changed into:

$$
\left\{\begin{array}{l}
x(l+1)=f[l, x(l), u(l)] \\
y(l)=g[l, x(l), u(l)]
\end{array}\right.
$$

At the $\mathrm{k}$ run process, it can be expressed as:

$$
\left\{\begin{array}{l}
x(l+1, k)=f[l, x(l, k), u(l, k)] \\
y(l, k)=g[l, x(l, k), u(l, k)]
\end{array}\right.
$$

The output error is :

$$
e(l, k)=y_{r}(l)-y(l, k)
$$

The discretized open-loop and closed-loop iterative learning control algorithms are as follows:

$$
\begin{aligned}
& u(l, k+1)=u(l, k)+K_{p} e(l, k)+K_{i} \sum_{j=0}^{l} e(j, k)+K_{d}[e(l, k)-e(l-1, k)] \\
& u(l, k+1)=u(l, k)+K_{p} e(l, k+1)+K_{i} \sum_{j=0}^{l} e(j, k+1)+K_{d}[e(l, k+1)-e(l-1, k+1)]
\end{aligned}
$$

Discreted open-loop and closed-loop general learning control algorithms are presented in the form of:

$$
\begin{aligned}
& u(l, k+1)=L[u(l, k), e(l, k)] \\
& u(l, k+1)=L[u(l, k), e(l, k+1)]
\end{aligned}
$$

Where $\mathrm{L}$ is linear or nonlinear operator.

The open-loop iterative learning control algorithm adopts the off-line calculation method, therefore the calculation requirements of the system are not high, which uses the output error of the previous system, so the control effect is not as good as closed-loop iterative control algorithm. The closed- loop iterative learning control requires on-line calculation, so it requires higher system.

\section{Design of Trajectory Control Model for Seabed Mining Vehicle based on Iterative Learning}

\section{Establishment of Trajectory Model in Polar Coordinates of Seabed Mining Vehicle}

Taking the seabed mining vehicle as the object of study, the trajectory model of the mining vehicle is set up as shown in Fig. 3.

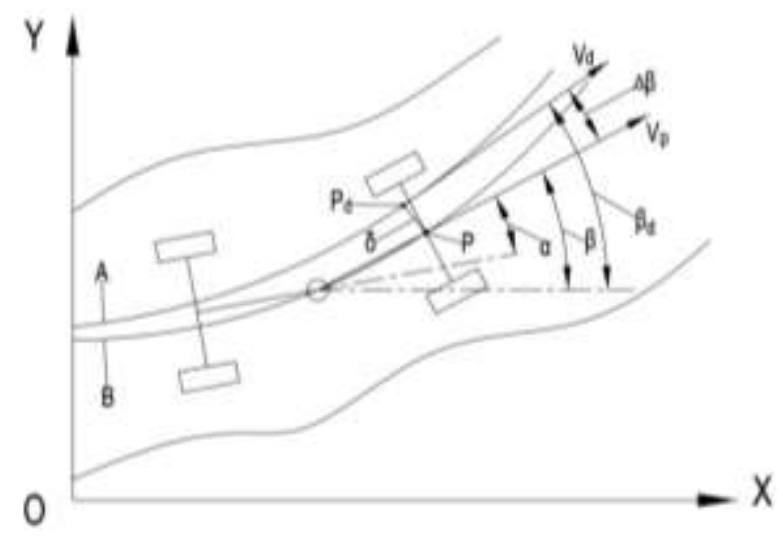

Figure 3. Trajectory Model of Seabed Mining Vehicle 


\section{Open Loop Iterative Learning Motion Trajectory Control Model}

Using the basic principle of the open-loop iterative learning control algorithm, the trajectory control in autonomous driving process is achieved. The steering angle $\alpha$ and the traveling speed $\mathrm{Vp}$ are selected as the input control variables, and two parameters, the deviation of the heading angle $\beta$ and the deviation of the motion trajectory $e$, are taken as the feedback correction quantities of the iterative learning, therefore, an open loop iterative learning motion trajectory control model is established. The block diagram of the open-loop iterative learning motion trajectory control model is shown in Fig. 4.

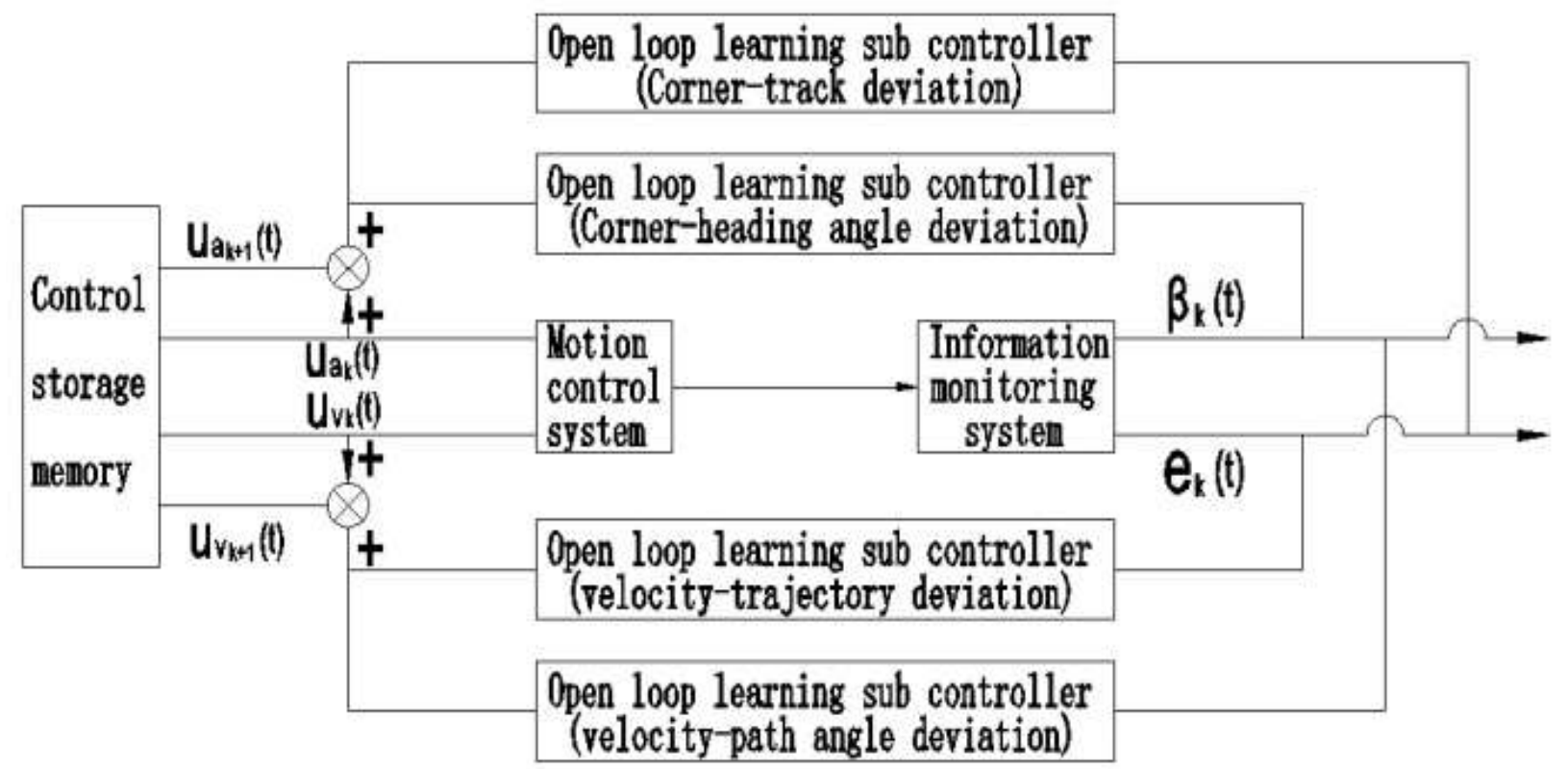

Figure 4. Open Loop Iiterative Learning Motion Trajectory Control Model Block Diagram

The open-loop iterative learning motion trajectory control model is designed as follows:

$$
\begin{aligned}
& u_{\alpha_{k+1}}(t)=u_{\alpha_{k}}(t)+K_{p 1} \beta_{k}(t)+K_{i 1} \int_{0}^{t} \beta_{k}(s) d s+K_{d 1} \frac{d \beta_{k}(t)}{d t}+K_{p 2} e_{k}(t)+K_{i 2} \int_{0}^{t} e_{k}(s) d s+K_{d 2} \frac{d e_{k}(t)}{d t} \\
& u_{v_{k+1}}(t)=u_{v_{k}}(t)+K_{p 3} \beta_{k}(t)+K_{i 3} \int_{0}^{t} \beta_{k}(s) d s+K_{d 3} \frac{d \beta_{k}(t)}{d t}+K_{p 4} e_{k}(t)+K_{i 4} \int_{0}^{t} e_{k}(s) d s+K_{d 4} \frac{d e_{k}(t)}{d t}
\end{aligned}
$$

Where $K_{p 1}, K_{i 1}, K_{d 1}$ are the parameters of an open-loop learning sub controller (corner-heading angle deviation); $K_{p 2}, K_{i 2}, K_{d 2}$ are the parameters of an open-loop learning sub controller(corner-trajectory deviation); $K_{p 3}, K_{i 3}, K_{d 3}$ are the parameters of an open-loop learning sub controller (velocity-path angle deviation); $K_{p 4}, K_{i 4}, K_{d 4}$ are the parameters of an open-loop learning sub controller (velocity-trajectory deviation); $u_{\alpha_{k+1}}(t), u_{v_{k+1}}(t)$ are the controlled variables of steering angle and traveling speed respectively in k+1 iterative learning; $u_{\alpha_{k}}(t) 、 u_{v_{k}}(t)$ are the controlled variables of steering angle and traveling speed respectively in the $\mathrm{k}$ iterative learning.

Considering that the computer control system adopts digital control, therefore, the discrete processing is carried out, and the discrete control model is as follows:

$$
\begin{aligned}
& u_{\alpha}(l, k+1)=u_{\alpha}(l, k)+K_{p 1} \beta(l, k)+K_{i 1} \sum_{j=0}^{l} \beta(j, k)+K_{d 1}[\beta(l, k)-\beta(l-1, k)]+K_{p 2} e(l, k)+K_{i 2} \sum_{j=0}^{l} e(j, k)+K_{d 2}[e(l, k)-e(l-1, k)] \\
& u_{v}(l, k+1)=u_{v}(l, k)+K_{p 3} \beta(l, k)+K_{i 3} \sum_{j=0}^{l} \beta(j, k)+K_{d 3}[\beta(l, k)-\beta(l-1, k)]+K_{p 4} e(l, k)+K_{i 4} \sum_{j=0}^{l} e(j, k)+K_{d 4}[e(l, k)-e(l-1, k)]
\end{aligned}
$$

\section{Closed Loop Iterative Learning Motion Trajectory Control Model}

The closed loop iterative learning control algorithm also selects the steering angle $\alpha$ and the traveling speed $\mathrm{Vp}$ as the input control quantities, at the same time, heading angle deviation $\beta$ and 
trajectory deviation $e$ are chosen as the feedback correction quantities of iterative learning, then a closed loop iterative learning trajectory control model is established. The block diagram of the closed loop iterative learning motion trajectory control model is shown in Fig. 5.

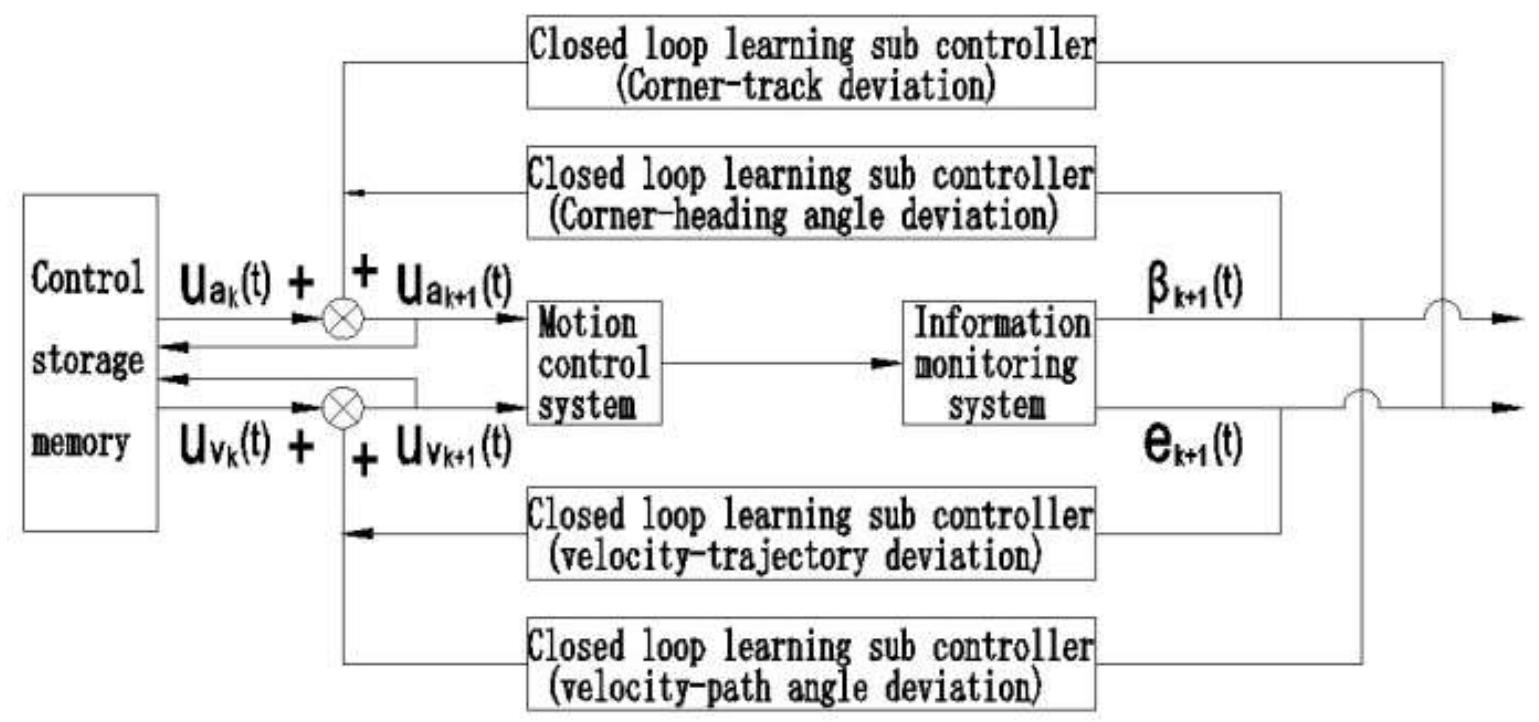

Figure 5. Block Diagram of Closed Loop Iterative Learning Trajectory Control Model

The closed-loop iterative learning motion trajectory control model is designed as follows:

$$
\begin{aligned}
& u_{\alpha_{k+1}}(t)=u_{\alpha_{k}}(t)+K_{p 1} \beta_{k+1}(t)+K_{i 1} \int_{0}^{t} \beta_{k+1}(s) d s+K_{d 1} \frac{d \beta_{k+1}(t)}{d t}+K_{p 2} e_{k+1}(t)+K_{i 2} \int_{0}^{t} e_{k+1}(s) d s+K_{d 2} \frac{d e_{k+1}(t)}{d t} \\
& u_{v_{k+1}}(t)=u_{v_{k}}(t)+K_{p 3} \beta_{k+1}(t)+K_{i 3} \int_{0}^{t} \beta_{k+1}(s) d s+K_{d 3} \frac{d \beta_{k+1}(t)}{d t}+K_{p 4} e_{k+1}(t)+K_{i 4} \int_{0}^{t} e_{k+1}(s) d s+K_{d 4} \frac{d e_{k+1}(t)}{d t}
\end{aligned}
$$

Where $K_{p 1}, K_{i 1}, K_{d 1}$ are the parameters of an closed-loop learning sub controller (corner-heading angle deviation); $K_{p 2}, K_{i 2}, K_{d 2}$ are the parameters of an closed-loop learning sub controller(corner-trajectory deviation); $K_{p 3}, K_{i 3}, K_{d 3}$ are the parameters of an closed-loop learning sub controller (velocity-path angle deviation); $K_{p 4}, K_{i 4}, K_{d 4}$ are the parameters of an closed-loop learning sub controller (velocity- trajectory deviation); $u_{\alpha_{k+1}}(t), u_{v_{k+1}}(t)$ are the controlled variables of steering angle and traveling speed respectively in $k+1$ iterative learning; $u_{\alpha_{k}}(t) 、 u_{v_{k}}(t)$ are the controlled variables of steering angle and traveling speed respectively in the $\mathrm{k}$ iterative learning.

Discretization is also performed, and the discrete control model is as follows:

$$
u_{\alpha}(l, k+1)=u_{\alpha}(l, k)+K_{p 1} \beta(l, k)+K_{i 1} \sum_{j=0}^{l} \beta(j, k+1)+K_{d 1}[\beta(l, k+1)-\beta(l-1, k+1)]+K_{p 2} e(l, k+1)+K_{i 2} \sum_{j=0}^{l} e(j, k+1)+K_{d 2}[e(l, k+1)-e(l-1, k+1)]
$$

$u_{v}(l, k+1)=u_{v}(l, k)+K_{p 3} \beta(l, k+1)+K_{i 3} \sum_{j=0}^{l} \beta(j, k+1)+K_{d 3}[\beta(l, k+1)-\beta(l-1, k+1)]+K_{p 4} e(l, k+1)+K_{i 4} \sum_{j=0}^{l} e(j, k+1)+K_{d 4}[e(l, k+1)-e(l-1, k+1)]$

\section{Conclusion}

Based on the basic principle of iterative learning control, an open-loop iterative learning control algorithm and a closed loop iterative learning control algorithm for controlling the trajectory of deep-sea mining vehicles are proposed. The mining vehicle runs repeatedly on the transport path, using the deviation of the heading angle and the trajectory deviation as the feedback correction in each iteration operation. The trajectory deviation of the desired trajectory is constantly corrected so 
that the mining vehicle can track the desired trajectory as accurately as possible, and the trajectory of the vehicle can be precisely controlled.

\section{Acknowledgments}

The work was the project supported by Scientific Research Projects of Weifang University (2012Z08).

\section{References}

[1] Arimoto S,Kawamura S,Miyazaki F. Bettering Operation of Robots by Learning. Journal of Robotic Systems, Vol. 1 No.2, 1984, PP.123-140.

[2] H. B. Wang, Y. Wang, Open-closed loop ILC corrected with anglerelationship of output vectors for tracking control of manipulator. Acta Automatica Sinica, Vol.36, No.12, December 2010, pp. 1758-1765.

[3] S.P.Li, M.X.Sui,S.Zhu, Iterative learning control with initial state learning for non-affine nonlinear systems, Journal of ZHEJIANG Universityof Tecnology, Vol. 38 No. 3, June 2010, pp.269-272.

[4] H. Zhang, D.Y. Luo, H.J. Huang, X. Luo, Fuzzy Iterative Learning Control of Robot and lts Simulation, Techniques of Automation and Application, No. 2, 2002, pp.3-5,32.

[5] W. Zhan, Gain Coefficient Variable Iterative Learning Control and it's Application for Robot, Micro Computer Applications, Vol.28, No.7, July 2007, pp. 678-681.

[6] S.L.Yan, H. Zhang, Y.L. Wang, X.P. Xiao, Trajectory tracking of mobile robot using iterative learning control in polar coordinates, Journal of Computer Applications, Vol.30, No. 8, August 2010, pp. 2017-2020.

[7] H.Lin, L.Wang, Iterative Learning Control Theory, $\mathrm{Xi}$ an: Northwestern Polytechnical University Press, 1998.

[8] S.L.Xie, S.P.Tian,Zh.D.Xie, The Theory and Application of IterativeLearning Control, Bei Jing: Science Press, 2005. 